\title{
Recent Advances in Therapeutics for B-Cell Lymphoma
}

\author{
Yasuhito Terui \\ Dept. of Hematology Oncology, Cancer Institute Hospital, Japanese Foundation for Cancer Research, Tokyo 135-8550 Japan
}

\begin{abstract}
Objectives: The molecular targeting drugs for the treatment of B-cell lymphoma have been dramatically developed. Recently, novel drugs of monoclonal antibodies and small molecules are approved by US FDA (Food and Drug Administration). Key Findings: This review summarizes characteristics, mechanisms, and results of the trials in the novel molecular targeting drugs for B-cell lymphoma such as obinutuzumab, polatuzumab vedotin, ibrutinib, idelalisib, and venetoclax. Summary: As a novel anti-CD20 antibody, obinutuzumab has been clinically developed on going. ADC (antibody drug conjugate) against CD79b molecules is also developed for B-cell lymphoma. BCR pathway is one of the most crucial pathways, and ibrutinib is a BTK (Bruton's tyrosine kinase) inhibitor that is under development for the treatment of B-cell malignancies, including CLL (chronic lymphocytic leukemia), MCL (mantle cell lymphoma), and DLBCL (diffuse large B-cell lymphoma), as well as FL (follicular lymphoma). BCL-2 family dysfunction and impairment of apoptosis are common in most B-cell lymphoid malignancies. Venetoclax, which is a highly selective BCL-2 inhibitor, a mimic for its BCL2 homolog 3-domain to induce apoptosis, is also reported to be active against B-cell malignancies. Conclusions: Mechanism-based combination regimens including these drugs may be required in the future.
\end{abstract}

Key words: obinutuzumab, polatuzumab vedotin, ibrutinib, idelalisib, venetoclax.

\section{Introduction}

In the past decade, the treatment for B-cell lymphoma has been developed largely. Rituximab, a chimeric monoclonal antibody that targets against CD20 molecule, is the first monoclonal antibody approved for the treatment of B-cell lymphoma 20 years ago [1]. It has been shown that rituximab exerts cytotoxic activities through $\mathrm{CDC}$ (complement-dependent cytotoxicity) and ADCC (antibody-dependent cell-mediated cytotoxicity), and that it directly induces apoptosis. The proven clinical efficacy and success of rituximab has led to development of the other anti-CD20 antibodies such as ofatumumab [2] and obinutuzumab [3] in recent years. Moreover, ADCs (antibody-drug conjugates) against CD22 and CD79b molecules are also developed for B-cell lymphoma [4]. On the other hands, the small molecule inhibitors targeting apoptosis pathways, proteasome, and other pathways of B-cell lymphoma are being developed as novel drugs for B-cell lymphoma. Especially, BCR (B-cell receptor) pathway

Corresponding author: Yasuhito Terui, M.D., Ph.D, director, research fields: hematology oncology. is one of the most crucial pathways for cell growth, anti-apoptosis of B-cell malignant cells [5]. Ibrutinib is a small molecule, once-daily, orally available, BTK (Bruton's tyrosine kinase) inhibitor [6] that is under development for the treatment of B-cell malignancies, including chronic lymphocytic leukemia, mantle cell lymphoma, and diffuse large B cell lymphoma, as well as follicular lymphoma. Here, I will discuss about development of novel drugs for B-cell lymphoma in the present and the future.

\section{Novel Drugs for B-Cell Lymphoma}

\subsection{Obinutuzumab}

Obinutuzumab is a glycoengineered type II anti-CD20 monoclonal antibody (mAb) currently approved for use in previously untreated CLL (chronic lymphocytic leukemia) in combination with chlorambucil, as well as for treatment of rituximab-refractory FL (follicular lymphoma) [7, 8]. Obinutuzumab is distinguished from rituximab by two molecular features. Absence of fucosylation at $\mathrm{Fc}$ portion in obinutuzumab results in enhanced ADCC activity [9], and type II-mediated mechanism of action 
of obinutuzumab is characterized by enhanced direct cell death induction [10].

Since preclinical studies and early phase studies of obinutuzumab monotherapy and combination therapy in B-cell malignancies have succeeded, obinutuzumab plus chemotherapy has been investigated in four phase 3 trials (Table 1).

The GALLIUM study was designed as a comparison of the efficacy and safety of obinutuzumab or rituximab plus chemotherapy followed by maintenance immunotherapy in previously untreated indolent NHL [11]. Obinutuzumab plus chemotherapy demonstrated a significantly reduced risk of progression or death relative to rituximab plus chemotherapy in FL (hazard ratio [HR] $0.66,95 \%$ CI [confidence interval] $0.51-0.85(p=0.001)$.

In the GADOLIN study, patients with rituximab-refractory indolent NHL were randomized to bendamustine monotherapy $\left(120 \mathrm{mg} / \mathrm{m}^{2}\right.$ for six cycles) or obinutuzumab plus bendamustine $\left(90 \mathrm{mg} / \mathrm{m}^{2}\right.$ for six cycles) followed by obinutuzumab maintenance
[12]. Updated results confirmed the PFS benefit and showed OS benefit in favor of obinutuzumab arm.

In the GOYA trial, previously untreated patients with DLBCL were randomized to obinutuzumab or rituximab every 21 days for eight cycles in combination with 6-8 cycles of CHOP-based chemotherapy [13]. Final analysis showed no significant difference in PFS between obinutuzumab and rituximab treatment arms. However, a trend toward benefit with obinutuzumab compared to rituximab was observed in the germinal center B-cell-like, but not the activated B-cell-like, patients.

In the CLL11 trial, patients previously untreated CLL were randomized to chlorambucil plus obinutuzumab or chlorambucil plus rituximab or chlorambucil alone for six cycles [14, 15]. Both combination therapies significantly improved PFS compared to chlorambucil alone. On the other hands, obinutuzumab arm, but not rituximab arm, significantly prolonged OS.

Table 1 Phase 3 trials of obinutuzumab in patients with B-cell NHL or CLL.

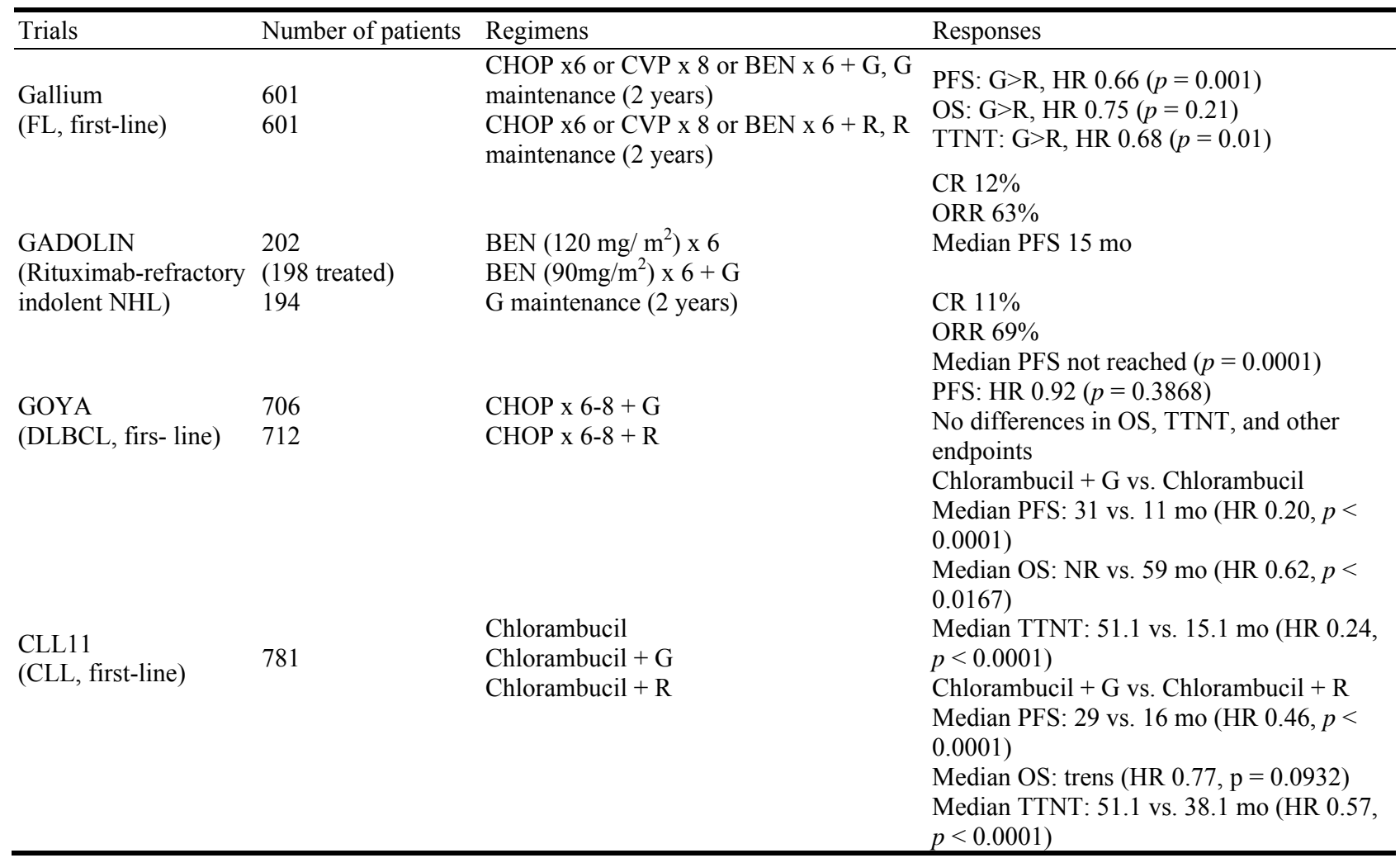


In the GALLIUM, GOYA, and CLL11, the risk of infusion-related reaction in cycle 1 seems to be higher with obinutuzumab than with rituximab, but the infusion-related reaction occurred with low and similar frequency in subsequent cycles

\subsection{Polatuzumab Vedotin}

A novel therapeutic approach is the introduction of ADCs (antibody drug conjugates) in which cytotoxic drugs are conjugated to antibodies against antigens expressed on tumor cells [16]. Recently, ADC has been shown to be effective in the treatment of malignant lymphoma. Brentuximab vedotin against CD30 achieved high response rates in patients with relapsed/refractory CD30-positive Hodgkin's lymphoma and anaplastic large-cell lymphoma $[17,18]$.

$\mathrm{CD} 22$ and CD79b are expressed in the vast majority of B cells and therefore represent promising targets for ADCs. Preclinical data for an anti-CD79b antibody conjugated to the microtubule-disrupting agent MMAE (monomethyl auristatin E) showed the efficacy in several B-cell lymphomas including DLBCL and FL [19-21].

Polatuzumab vedotin is an anti-CD79b monoclonal antibody conjugated to MMAE. In a phase I study, this drug was administrated at escalating dose from 0.1 to $2.4 \mathrm{mg} / \mathrm{kg}$ for $34 \mathrm{NHL}$ and 18 CLL patients [22]. The recommended dose for phase 2 trial was $2.4 \mathrm{mg} / \mathrm{kg}$ as a single agent and in combination with rituximab. On the other hands, MTD in CLL was $1.0 \mathrm{mg} / \mathrm{kg}$ due to DLT. In the phase 2 of the study, 34 NHL patients to the single agent arm and nine NHL patients to the rituximab combination arm were enrolled. The most common Grade 3-4 AEs (adverse events) in the single-agent arm were neutropenia (40\%), anemia (11\%), and sensory neuropathy (9\%). In the combination therapy, $77 \%$ of patients showed grade 3-4 AEs including neutropenia, febrile neutropenia, and anemia. Objective and durable responses were observed in 23 of 42 patients (54.8\%) with NHL in the single-agent arm and seven of nine (77.8\%) in the combination arm. In CLL patients, no responses were observed. Moreover, Morschhauser et al. [23] reported the results of a phase 2 randomized trial of polatuzumab vedotin and rituximab for patients with relapsed/refractory NHL. Fifty-nine patients received polatuzumab vedotin $(2.4 \mathrm{mg} / \mathrm{kg})$ plus rituximab $\left(375 \mathrm{mg} / \mathrm{m}^{2}\right.$ ) every 21 days. Patients included 39 with DLBCL and 20 with FL. An additional 20 patients received polatuzumab vedotin $(1.8 \mathrm{mg} / \mathrm{kg})$ plus rituximab $\left(375 \mathrm{mg} / \mathrm{m}^{2}\right)$ every 21 days in nonrandomized cohort. The overall response rate (ORR) in DLBCL was $56 \%$ with $15 \%$ of CR (complete response) rate, and median PFS was 5.4 months. The ORR in FL was $70 \%$ with $40 \%$ of CR rate, and median PFS was not reached. The patients receiving polatuzumab vedotin at dose of $1.8 \mathrm{mg} / \mathrm{kg}$ showed only PR in seven cases (44\%). The most common AEs in polatuzumab vedotin arm included fatigue (55\%), diarrhea (43\%), nausea (37\%), peripheral neuropathy (39\%), neutropenia (27\%), constipation (26\%), sensory neuropathy (25\%), and decreased appetite (25\%). Serious AEs were reported $36 \%$ in polatuzumab vedotin arm. Treatment discontinuation was reported in $41 \%$ of patients, mostly due to peripheral neuropathy with median time of 5.6 months.

\subsection{BCR Pathway-Targeted Drugs (Small Molecules)}

The BCR (B-cell receptor) has been recognized as an important pathway in B cell development as well as B-cell lymphomas (Fig. 1) [24]. Various approaches for the treatments have been tried to target this pathway. Regarding these approaches, targeting Syk [25], phosphatidyl-inositol-3 kinase delta (PI3K delta) [26], and BTK (Bruton's tyrosine kinase) showed preclinical and clinical activities such as the BTK inhibitor ibrutinib and the PI3K inhibitor idelalisib.

\subsection{BTK Inhibitor Ibrutinib}

Ibrutinib is the first-in-class orally bioactive small molecule inhibitor of BTK and acts by forming a stable 


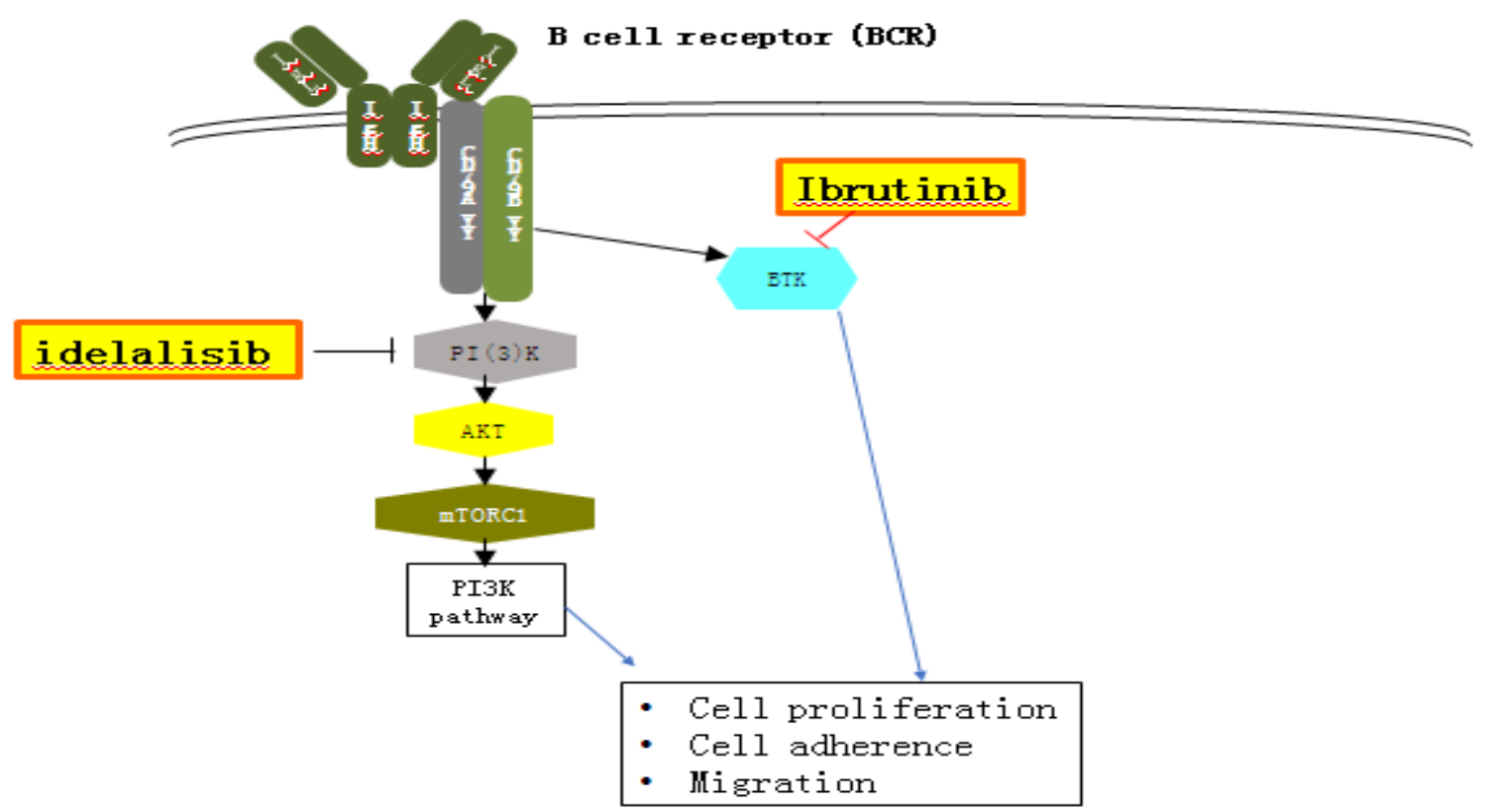

Fig. 1 BCR signaling pathway and their inhibitors.

covalent bond to the amino acid cysteine at position 481 in the BTK active site [27]. Although ibrutinib shows a short half-life, the covalent binding nature and high selectivity allows a once-daily dosing with a complete occupancy of the BTK active site in peripheral mononuclear cells [28]. Moreover, BTK activation plays a major role in other B cell lymphomas as well as CLL.

MCL (mantle cell lymphoma) is a rare subtype of non-Hodgkin's B-cell lymphoma characterized by the translocation $[11,14]$ with Cyclin D1 activation. MCL often shows an aggressive clinical course with poor prognosis. Younger patients $(<65$ years old $)$ are treated with intensive chemotherapy such as R-hyper CVAD/MA regimen followed by autologous stem cell transplantation [29]. Elderly patients were commonly treated with more tolerable immunochemotherapies such as bendamustine plus rituximab [30, 31], VR-CAP (bortezomib, rituximab, cyclophosphamide, doxorubicin, and prednisolone) with rituximab maintenance as front-line therapy as frontline therapy [32]. In spite of high OOR with these frontline therapies, prognosis of most MCL patients is not improved so far. Therefore, since pathogenesis of MCL is biologically related to chronic BCR signaling, BTK inhibitor was tested in this lymphoma. Data of a phase 1 study for ibrutinb in relapsed/refractory MCL patients showed high efficacy (28). A phase 2 study in $111 \mathrm{relapsed} / \mathrm{refractory} \mathrm{MCL}$ patients showed OOR of $68 \%$ with $21 \% \mathrm{CR}$ and median PFS of 13.9 months [33]. Finally, a phase 3 trial compared ibrutinib monotherapy with temsilorimus monotherapy [34]. Ibrutinib showed better tolerability and significant higher response rates in relapsed/refractory MCL patients (ORR $72 \%$ versus $40 \%, p<0.001$ ). Median PFS was significantly prolonged (14.6 vs. 6.2 months, $p<0.0001)$.

Combination with ibrutinib and rituximab showed response in almost all patients with non-blastoid MCL with low Ki67 index, whereas early relapses were frequently seen in patients with aggressive disease [35].

Nodal Marginal zone lymphoma (MZL) is a rare indolent lymphoma. Since Nodal MZL is small population and heterogeneous, clinical study are few and mostly small. Therefore, patients are treated as patients with indolent lymphoma groups. MZL is also associated with chronic inflammation, leading to 
continuous B cell stimulation by BCR signaling. A phase 2 trial for single-agent ibrutinib in patients with relapsed/refractory MZL showed high efficacy by disrupting the BCR signaling with ibrutinib [36]. Patients with at least one prior anti-CD20-based therapy showed ORR of $51 \%$ with median duration of response of 19 months. FDA approved ibrutinib for MZL by this data in January 2017.

\subsection{PI3K Inhibitor Idelalisib}

Another critical molecule for BCR pathway is PI3K in survival of CLL and FL cells. Idelalisib is a first-in-class, reversible, potent, selective $\mathrm{PI} 3 \mathrm{~K} \partial$ inhibitor [37], and induces cell cycle arrest in G0/G1 phase inhibiting proliferation and apoptosis in B-cell by caspase activation.

A phase 1 study of single-agent idelalisib in 54 patients with relapsed/refractory CLL showed ORR of $75 \%$ and median PFS of 15.8 months [38]. From this result, a phase 3 study was conducted as a randomized, double-blind, placebo-controlled trial on 220 patients with previously treated CLL. The patients, including high-risk patients with del17p, TP53 mutation, or IGHV lack, were randomized to receive rituximab pus idelalisib (150 mg BID) or placebo twice daily. The PFS in idelalisib versus placebo at 24 weeks was $93 \%$ versus $46 \%(p<0.001)$, and OS at 12 months was $92 \%$ versus $80 \%(p=0.02)$, respectively [26].

The role of single-agent idelalisib in relapsed indolent NHL was investigated in a phase 2 trial with 125 patients including FL $(n=72)$, SLL $(n=28), M Z L$ $(\mathrm{n}=15)$, and lymphoplasmacytic lymphoma $(\mathrm{n}=10)$. The ORR was $57 \%$, and the median duration of response was 12.5 months. The median PFS and OS were 11 months and 20.3 months, respectively (Table 2) [39].

The AEs related to idelalisib combining three most recent clinical studies are listed in Table 3 [26, 39, 40]. In the relapsed CLL study, the most common AEs in the idelalisib plus rituximab arm were fatigue (24\%), nausea (24\%), diarrhea (19\%), fever (29\%), and chills $(22 \%)$. SAEs (Serious AEs) included pneumonia (6\%), febrile neutropenia $(5 \%)$, diarrhea $(3 \%)$, pyrexia $(6 \%)$, and dyspnea (1\%). Laboratory abnormalities included elevation in alanine transaminase/aspartate transaminase, mostly occurring within the first 12 weeks of the treatment. Others were anemia, neutropenia, thrombocytopenia, and lymphocytosis.

In relapsed/refractory indolent NHL study, the most common AEs with single-agent idelalisib included diarrhea (13\%) and pneumonia (7\%). Dose reduction from $150 \mathrm{mg}$ BID to $100 \mathrm{mg}$ BID was required in 34\% of indolent NHL patients due to AEs such as hepatotoxicity, colitis, and pneumonia [40].

\subsection{Anti-apoptosis Molecule Mimetic Drug}

Apoptosis is an evolutionarily conserved process of cell death which plays a critical role in homeostasis [41]. There are two different apoptosis pathways: the "extrinsic pathway" and the "intrinsic pathway". The intrinsic pathway is triggered by a range of signals, such as DNA damage, growth factor-deprivation, and the release of apoptogenic proteins, including cytochrome $c$. BCL-2 gene product was the first anti-apoptosis protein discovered as fusion gene of $\mathrm{t}$ $[14,18]$ chromosomal translocation, which is the hallmark of FL [42]. Thereafter, more than 20 of BCL-2 family members have been identified so far.

Table 2 Efficacy of idelalisib in patients with previously treated indolent NHL.

\begin{tabular}{ll}
\hline Outcomes & Indolent NHL (previously treated, $\mathrm{n}=125)$ \\
\hline Median PFS (months) & 11 \\
Overall response rate & $57 \%$ \\
Complete response & $6 \%$ \\
Partial response & $50 \%$ \\
Lymph node response & $90 \%$ \\
OS (12 months) & $80 \%$ \\
\hline
\end{tabular}


Table 3 Adverse events and laboratory abnormalities of idelalisib.

\begin{tabular}{|c|c|c|c|c|c|c|c|c|}
\hline \multirow[t]{2}{*}{ AEs } & \multicolumn{2}{|c|}{ Idelalisib $+R(n=110)$} & \multicolumn{2}{|c|}{$\begin{array}{l}\text { Idelalisib+ placebo } \\
(\mathrm{n}=107)\end{array}$} & \multicolumn{2}{|c|}{ CLL front-line $(n=64)$} & \multicolumn{2}{|c|}{$\begin{array}{l}\text { Indolent NHL previously treated } \\
\qquad(\mathrm{n}=125)\end{array}$} \\
\hline & Any grade & Grade $\geq 3$ & Any grade & Grade $\geq 3$ & Any grade & Grade $\geq 3$ & Any grade & Grade $\geq 3$ \\
\hline Pyrexia & $32(29)$ & $3(3)$ & $17(16)$ & $1(1)$ & $27(42)$ & $2(3)$ & $35(28)$ & $2(2)$ \\
\hline Fatigue & $26(24)$ & $3(3)$ & $29(27)$ & $2(2)$ & $20(31)$ & 0 & $37(30)$ & $2(2)$ \\
\hline Nausea & $26(24)$ & 0 & $23(21)$ & 0 & $24(38)$ & $1(2)$ & $37(30)$ & $2(2)$ \\
\hline Chills & $24(22)$ & $2(2)$ & $17(16)$ & 0 & $23(36)$ & 0 & - & - \\
\hline Diarrhea & $21(19)$ & $4(4)$ & $15(14)$ & 0 & $25(39)$ & $11(17)$ & $54(43)$ & $16(13)$ \\
\hline $\begin{array}{l}\text { Infusion-related } \\
\text { reaction }\end{array}$ & $117(15)$ & 0 & $30(28)$ & $4(4)$ & - & - & NA & NA \\
\hline Cough & $16(15)$ & 0 & $27(25)$ & $2(2)$ & $21(33)$ & $1(2)$ & $36(29)$ & 0 \\
\hline Constipation & $13(12)$ & 0 & $12(11)$ & 0 & $11(17)$ & 0 & - & - \\
\hline Decreased appetite & $13(12)$ & 0 & $9(8)$ & $1(1)$ & - & - & $22(18)$ & $1(1)$ \\
\hline Vomiting & $13(12)$ & 0 & $8(7)$ & 0 & $14(22)$ & $2(3)$ & $19(15)$ & $3(2)$ \\
\hline Dyspnea & $12(11)$ & $2(2)$ & $10(19)$ & $3(3)$ & $16(25)$ & $4(6)$ & $22(18)$ & $4(3)$ \\
\hline Night sweats & $11(10)$ & 0 & $8(7)$ & 0 & $10(16)$ & 0 & $14(11)$ & 0 \\
\hline Rash & $11(10)$ & $2(2)$ & $6(6)$ & 0 & $37(58)$ & $8(13)$ & $16(13)$ & $2(2)$ \\
\hline AST/ALT elevation & $38(35)$ & $6(5)$ & $20(19)$ & $1(1)$ & $43(67)$ & $15(23)$ & $59(47) / 44(35)$ & $16(15) / 10(8)$ \\
\hline Anemia & $28(25)$ & $6(5)$ & $32(30)$ & $1514)$ & $15(23)$ & $2(3)$ & $35(28)$ & $2(2)$ \\
\hline Neutropenia & $60(55)$ & $37(34)$ & $52(49)$ & $24(22)$ & $34(53)$ & $18(28)$ & $70(56)$ & $34(27)$ \\
\hline Thrombocytopenia & $19(17)$ & $11(10)$ & $28(26)$ & $17(16)$ & $9(14)$ & $1(2)$ & $32(26)$ & $8(6)$ \\
\hline
\end{tabular}

The anti-apoptotic proteins such as BCL-2, BCL-X $\mathrm{L}_{\mathrm{L}}$, BCL-W, BFL-1/A1, and MCL-1 have four BH (BCL-2 homology) domains and interact with other BCL-2 family proteins to prevent mitochondrial outer membrane permeabilization, which is the key point of "no return" in the intrinsic pathway.

\subsection{Venetoclax}

Venetoclax was developed as a highly BCL-2-selective $\mathrm{BH} 3$-mimetic. It has a strong affinity only for BCL-2, with 100-fold less affinity for other BCL-2 family such as BCL-X $\mathrm{L}_{\mathrm{L}}$ or BCL-W. As well as navitoclax, which is another $\mathrm{BH} 3-$ mimetic and inhibits not only BCL-2 but also BCL-X $\mathrm{L}_{\mathrm{L}}$ or $\mathrm{BCL}-\mathrm{W}$, in vitro cytotoxicity of venetoclax is dependent on the presence of BAX and BAK, which in turn leads to cell death by apoptosis [43].

Venetoclax was first tested in relapsed/refractory CLL patients. It induced lymphocyte reduction in a dose-dependent manner without thrombocytopenia [44]. The first clinical trial of venetoclax was a phase 1 dose escalation study that enrolled 116 patients with relapsed/refractory CLL or SLL (small lymphocytic lymphoma), of which $92(79 \%)$ had a response and 20\% achieved CR, including 5\% with MRD negative as assessed by flow cytometry. Objective responses were common among patients with resistance to fludarabine with del17p and unmutated IGHV. PFS estimated for the $400 \mathrm{mg}$ dose groups was $69 \%$ (Table 4) [44]. In Phase 2, single-arm, multicenter study of venetoclax conducted exclusively in CLL patients with del17p, 107 patients were enrolled [45]. ORR determined by an independent review was achieved in $85(79.4 \%)$ patients with a median follow-up of 12.1 months. Response rates were similar regardless of refractory to prior fludarabine-based therapy. The clinical responses of CLL patients were also independent of del17p, TP53 mutation, and TP53 function [46]. Recently, a phase 2, open label, two-arm study reported the outcome of single-agent venetoclax in 64 CLL patients who relapsed after, or were refractory to ibrutinib or idelalisib, of whom 43 were on prior ibrutinib (Arm A) and 21 on prior idelalisib (Arm B). ORR by an independent review for Arm A 
Table 4 Clinical trials of single-agent venetoclax in CLL and B-cell malignancies.

\begin{tabular}{|c|c|c|c|c|}
\hline Diseases & Phase & Number of patients & ORR & $\mathrm{CR}+\mathrm{CRi}$ \\
\hline $\mathrm{R} / \mathrm{R}$ CLL & 1 & 116 & $79 \%$ & $20 \%$ \\
\hline $\begin{array}{l}\mathrm{R} / \mathrm{R} \text { CLL } \\
\text { with del17p }\end{array}$ & 2 & 107 & $79 \%$ & $12.1 \%$ \\
\hline $\mathrm{R} / \mathrm{R} \mathrm{CLL}$ & 2 & 120 & $\begin{array}{l}70 \% \text { (post-ibrutinib) } \\
48 \% \text { (post-idelalisib) }\end{array}$ & Not reported \\
\hline R/R NHL & 1 & 106 & $\begin{array}{l}18 \%(\mathrm{DLBCL}, 6 / 34) \\
35 \%(\mathrm{FL}, 11 / 29)\end{array}$ & Not reported \\
\hline
\end{tabular}

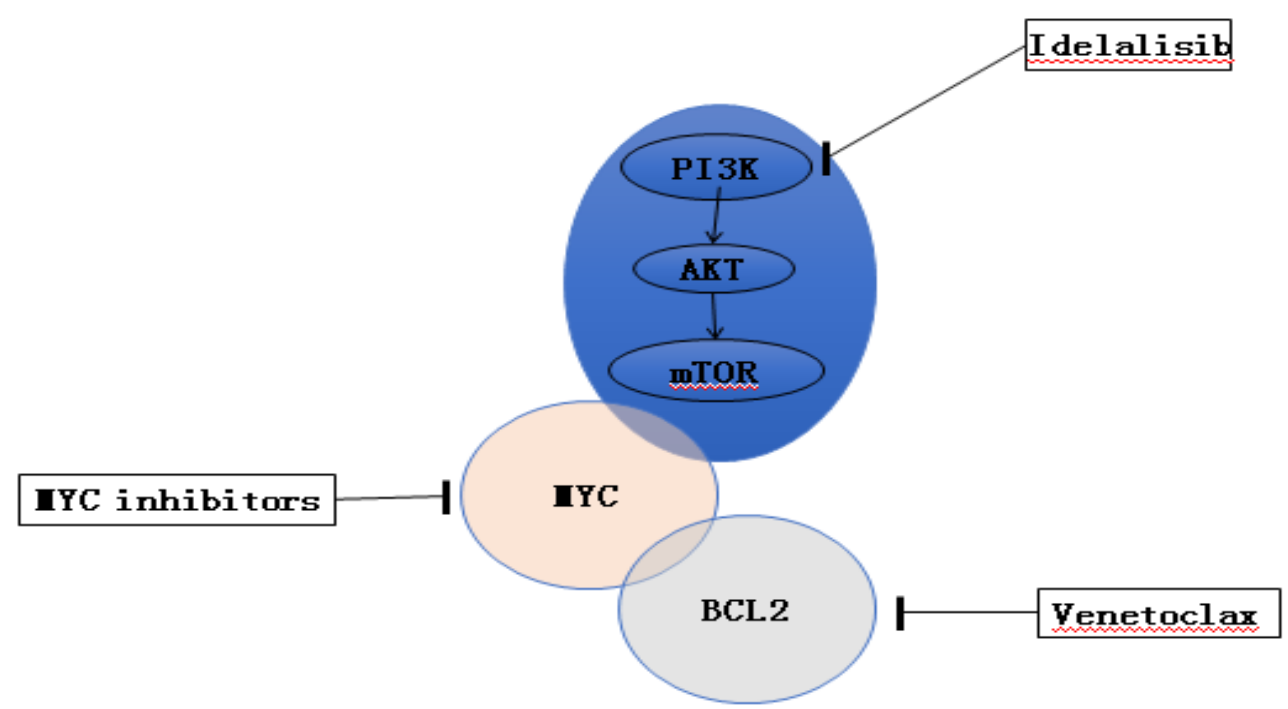

Fig. 2 Developing rational combination therapies based on biological knowledge in DLBCL.

and Arm B was $70 \%$ and $48 \%$, respectively. At the time of analysis, neither the PFS nor the OS has been reached. The estimated 12-month PFS was 72\%, and OSl was $90 \%$ for the overall group. This study demonstrated that venetoclax had strong activity in CLL patients who progressed during or after treatment with ibrutinib or idelalisib (Table 4) [47].

Single-agent venetoclax was also investigated in a phase 1 study in patients with relapsed/refractory NHL. The ORR varied in the different histologic subgroups, with the highest rates observed in WM $(100 \%$ including all PR) and MCL (75\% including 21\% CR). Responses were less frequent in FL (ORR: 38\%, CR: 14\%) and DLBCL (ORR: 18\%, CR: 12\%), and the median duration of response of DLBCL was only 3.3 months (Table 4) [48].

\section{Future}

Recently, many molecular targeting drugs have been developed so far and will be done in the future. However, these drugs need to be selected to match each patient or each disease. For instance, DLBCL represents the most common type of non-Hodgkin lymphoma. Although histologically well defined, DLBCL is heterogeneous at the molecular level. Patients with DLBCL have variable response to standard RCHOP therapy, which cures about $50 \%$ of the patients. There is robust evidence for oncogenic cooperation between MYC, BCL2, and activated PI3K signaling pathway in lymphomagenesis, providing an opportunity for developing mechanism-based therapy for $\mathrm{MYC}+/ \mathrm{BCL} 2+\mathrm{DLBCL}$ (Fig. 2) [49]. Mechanism-based combination regimens including these drugs may be required for a future direction.

\section{Conclusions}

Now, many molecular targeting drugs for the treatment of B-cell lymphoma are available in clinical 
practice. In the future, mechanism-based combination regimens including these drugs may be required for the treatment of B-cell malignancies.

\section{References}

[1] Zappasodi, R., de Braud, F., and Di Nicola, M. 2015. "Lymphoma Immunotherapy: Current Status." Front Immunol. 1 (6): 448.

[2] Coiffier, B., Lepretre, S., Pedersen, L. M., Gadeberg, O., Fredriksen, H., van Oers, M. H., et al. 2008. "Safety and Efficacy of Ofatumumab, a Fully Human Monoclonal Anti-CD20 Antibody, in Patients with Relapsed or Refractory B-Cell Chronic Lymphocytic Leukemia: A Phase 1-2 Study." Blood 111 (3): 1094-100.

[3] Niederfellner, G., Lammens, A., Mundigl, O., Georges, G. J., Schaefer, W., Schwaiger, M., et al. 2011. "Epitope Characterization and Crystal Structure of GA101 Provide Insights into the Molecular Basis for Type I/II Distinction of CD20 Antibodies." Blood 118 (2): 358-67.

[4] Hamblett, K. J., Senter, P. D., Chace, D. F., Sun, M. M., Lenox, J., Cerveny, C. G., et al. 2004. "Effects of Drug Loading on the Antitumor Activity of a Monoclonal Antibody Drug Conjugate." Clin Cancer Res. 10 (20): 7063-70.

[5] Smith, C. I., Baskin, B., Humire-Greiff, P., Zhou, J. N., Olsson, P. G., Maniar, H. S., et al. 1994. "Expression of Bruton's Agammaglobulinemia Tyrosine Kinase Gene, BTK, Is Selectively Down-Regulated in T Lymphocytes and Plasma Cells.” J. Immunol. 152 (2): 557-65.

[6] Ponader, S., and Burger, J. A. 2014. "Bruton's Tyrosine Kinase: From X-Linked Agammaglobulinemia toward Targeted Therapy for B-Cell Malignancies.” J. Clin. Oncol. 32 (17): 1830-9.

[7] GAZYVA®. US prescribing information. Cited 2017 Apr. Available http://www.accessdata.fda.gov/drugsatfda_docs/label/201 5/125486s 10lbeldt.pdf.

[8] GAZYVARO ${ }^{\circledR}$. Summary of product characteristics. [cited 2017 Apr.] Available at: http://www.ema.europa.eu/docs/en_GB/document_library /EPAR__Product_Information/human/002799/WC50017 1594.pdf.

[9] Mössner, E., Brünker, P., Moser, S., Püntener, U., Schmidt, C., Herter, S., et al. 2010. "Increasing the Efficacy of CD20 Antibody Therapy through the Engineering of a New Type II Anti-CD20 Antibody with Enhanced Direct and Immune Effector Cell-Mediated B-Cell Cytotoxicity." Blood 115 (22): 4393-402.

[10] Klein, C., Lammens, A., Schäfer, W., Georges, G., Schwaiger, M., Mössner, E., et al. 2013. "Epitope Interactions of Monoclonal Antibodies Targeting CD20 and Their Relationship to Functional Properties." MAbs. 5 (1): 22-33.

[11] Marcus, R., Davies, A., Ando, K., Klapper, W., Opat, S., Owen, C., et al. 2017. "Obinutuzumab for the First-Line Treatment of Follicular Lymphoma.” N. Engl. J. Med. 377 (14): 1331-44.

[12] Sehn, L. H., Chua, N., Mayer, J., Dueck, G., Trněný, M., Bouabdallah, K., et al. 2016. "Obinutuzumab plus Bendamustine versus Bendamustine Monotherapy in Patients with Rituximab-Refractory Indolent Non-Hodgkin Lymphoma (GADOLIN): A Randomised, Controlled, Open-Label, Multicentre, Phase 3 Trial." Lancet Oncol. 17 (8): 1081-93.

[13] Vitolo, U., Trněný, M., Belada, D., Burke, J. M., Carella, A. M., Chua, N., et al. 2017. "Obinutuzumab or Rituximab plus Cyclophosphamide, Doxorubicin, Vincristine, and Prednisone in Previously Untreated Diffuse Large B-Cell Lymphoma.” J. Clin. Oncol. 35 (31): 3529-37.

[14] Goede, V., Fischer, K., Busch, R., Engelke, A., Eichhorst, B., Wendtner, C. M., et al. 2014. "Obinutuzumab plus Chlorambucil in Patients with CLL and Coexisting Conditions.” N. Engl. J. Med. 370 (12): 1101-10.

[15] Goede, V., Fischer, K., Engelke, A., Schlag, R., Lepretre, S., Montero, L. F., et al. 2015. "Obinutuzumab as Frontline Treatment of Chronic Lymphocytic Leukemia: Updated Results of the CLL11 Study." Leukemia 29 (7): 1602-4.

[16] Leslie, L. A., and Younes, A. 2013. Antibody-Drug Conjugates in Hematologic Malignancies. Am Soc Clin Oncol Educ Book. pp.108-13.

[17] Younes, A., Bartlett, N. L., Leonard, J. P., Kennedy, D. A., Lynch, C. M., Sievers, E. L., Forero-Torres, A. 2010. "Brentuximab Vedotin (SGN-35) for Relapsed CD30-Positive Lymphomas." N. Engl. J. Med. 363 (19): 1812-21.

[18] Pro, B., Advani, R., Brice, P., Bartlett, N. L., Rosenblatt, J. D., Illidge, T., et al. 2012. "Brentuximab Vedotin (SGN-35) in Patients with Relapsed or Refractory Systemic Anaplastic Large-Cell Lymphoma: Results of a Phase II Study.” J. Clin. Oncol. 30 (18): 2190-6.

[19] Polson, A. G., Yu, S. F., Elkins, K., Zheng, B., Clark, S., Ingle, G. S., et al. 2007. "Antibody-Drug Conjugates Targeted to CD79 for the Treatment of Non-Hodgkin Lymphoma.” Blood 110 (2): 616-23.

[20] Dornan, D., Bennett, F., Chen, Y., Dennis, M., Eaton, D., Elkins, K., et al. 2009. "Therapeutic Potential of an Anti-CD79b Antibody-Drug Conjugate, Anti-CD79b-vc-MMAE, for the Treatment of Non-Hodgkin Lymphoma.” Blood 114 (13): 2721-9

[21] Polson, A. G., Williams, M., Gray, A. M., Fuji, R. N., Poon, K. A., McBride, J., et al. 2012. 
"Anti-CD22-MCC-DM1: An Antibody-Drug Conjugate with a Stable Linker for the Treatment of Non-Hodgkin's Lymphoma." Leukemia 24 (9): 1566-73.

[22] Palanca-Wessels, M. C., Czuczman, M., Salles, G., Assouline, S., Sehn, L. H., Flinn, I., et al. 2015. "Safety and Activity of the Anti-CD79B Antibody-Drug Conjugate Polatuzumab Vedotin in Relapsed or Refractory B-Cell Non-Hodgkin Lymphoma and Chronic Lymphocytic Leukaemia: A Phase 1 Study." Lancet Oncol. 16 (6): 704-15.

[23] Morshhauser, F., Flinn, I., Advani, R. H., et al. 2014. "Preliminary Results of a Phase II Randomized Study (ROMULUS) of Polatuzumabvedotin or Pinatuzumab Vedotin plus Rituximab in Patients with Relapsed/Refractory Non-Hodgkin Lymphoma." Blood 124: 4457.

[24] Chiorazzi, N., Rai, K. R., Ferrarini, M. 2005. "Chronic Lymphocytic Leukemia.” N. Engl. J. Med. 352 (8): 804-15.

[25] Sharman, J., Hawkins, M., Kolibaba, K., Boxer, M., Klein, L., Wu, M., Hu, J., Abella, S., Yasenchak, C. 2015. “An Open-Label Phase 2 Trial of Entospletinib (GS-9973), a Selective Spleen Tyrosine Kinase Inhibitor, in Chronic Lymphocytic Leukemia.” Blood 125 (15): 2336-43.

[26] Furman, R. R., Sharman, J. P., Coutre, S. E., Cheson, B. D., Pagel, J. M., Hillmen, P., et al. 2014. "Idelalisib and Rituximab in Relapsed Chronic Lymphocytic Leukemia." N. Engl. J. Med. 370 (11): 997-1007.

[27] Honigberg, L. A., Smith, A. M., Sirisawad, M., Verner, E., Loury, D., Chang, B., et al. 2010. "The Bruton Tyrosine Kinase Inhibitor PCI-32765 Blocks B-Cell Activation and Is Efficacious in Models of Autoimmune Disease and B-Cell Malignancy." Proc Natl Acad Sci USA 107 (29): 13075-80.

[28] Advani, R. H., Buggy, J. J., Sharman, J. P., Smith, S. M., Boyd, T. E., Grant, B., et al. 2013. "Bruton Tyrosine Kinase Inhibitor Ibrutinib (PCI-32765) Has Significant Activity in Patients with Relapsed/Refractory B-Cell Malignancies.” J. Clin. Oncol. 31 (1): 88-94.

[29] Hermine, O., Hoster, E., Walewski, J., Bosly, A., Stilgenbauer, S., Thieblemont, C., et al. 2016. "European Mantle Cell Lymphoma Network. "Addition of High-Dose Cytarabine to Immunochemotherapy before Autologous Stem-Cell Transplantation in Patients Aged 65 Years or Younger with Mantle Cell Lymphoma (MCL Younger): A Randomised, Open-Label, Phase 3 Trial of the European Mantle Cell Lymphoma Network." Lancet 388 (10044): 565-75.

[30] Rummel, M. J., Niederle, N., Maschmeyer, G., Banat, G. A., von Grünhagen, U., Losem, C., et al. 2013. "Study Group Indolent Lymphomas (StiL). Bendamustine plus Rituximab versus CHOP plus Rituximab as First-Line
Treatment for Patients with Indolent and Mantle-Cell Lymphomas: An Open-Label, Multicentre, Randomised, Phase 3 Non-inferiority Trial." Lancet 381 (9873): 1203-10.

[31] Flinn, I. W., van der Jagt, R., Kahl, B. S., Wood, P., Hawkins, T. E., Macdonald, D., et al. 2014. "Randomized Trial of Bendamustine-Rituximab or R-CHOP/R-CVP in First-Line Treatment of Indolent NHL or MCL: The BRIGHT Study.” Blood 123 (19): 2944-52.

[32] Kluin-Nelemans, H. C., Hoster, E., Hermine, O., Walewski, J., Trneny, M., Geisler, C. H., et al. 2012. "Treatment of Older Patients with Mantle-Cell Lymphoma.” N. Engl. J. Med. 367 (6): 520-31.

[33] Wang, M. L., Rule, S., Martin, P., Goy, A., Auer, R., Kahl, B. S., et al. 2013. "Targeting BTK with Ibrutinib in Relapsed or Refractory Mantle-Cell Lymphoma.” N. Engl. J. Med. 369 (6): 507-16.

[34] Dreyling, M., Jurczak, W., Jerkeman, M., Silva, R. S., Rusconi, C., Trneny, M., et al. 2016. "Ibrutinib versus Temsirolimus in Patients with Relapsed or Refractory Mantle-Cell Lymphoma: An International, Randomised, Open-Label, Phase 3 Study." Lancet 387 (10020): 770-8.

[35] Wang, M. L., Lee, H., Chuang, H., Wagner-Bartak, N., Hagemeister, F., Westin, J., et al. 2016. "Ibrutinib in Combination with Rituximab in Relapsed or Refractory Mantle Cell Lymphoma: A Single-Centre, Open-Label, Phase 2 Trial." Lancet Oncol. 17 (1): 48-56.

[36] Noy, A., de Vos, S., Thieblemont, C., Martin, P., Flowers, C. R., Morschhauser, F., Collins, G. P., et al. 2017. "Targeting Bruton Tyrosine Kinase with Ibrutinib in Relapsed/Refractory Marginal Zone Lymphoma.” Blood 129 (16): 2224-32.

[37] Markham, A. 2014. "Idelalisib: First Global Approval." Drugs 74 (14): 1701-7.

[38] Brown, J. R., Byrd, J. C., Coutre, S. E., Benson, D. M., Flinn, I. W., Wagner-Johnston, N. D., et al. 2014. "Idelalisib, an Inhibitor of Phosphatidylinositol 3-Kinase p1108, for Relapsed/Refractory Chronic Lymphocytic Leukemia.” Blood 123 (22): 3390-7.

[39] Gopal, A. K., Kahl, B. S., de Vos, S., Wagner-Johnston, N. D., Schuster, S. J., Jurczak, W. J., Flinn, I. W., et al. 2014. "PI3K $\delta$ Inhibition by Idelalisib in Patients with Relapsed Indolent Lymphoma." N. Engl. J. Med. 370 (11): 1008-18.

[40] O'Brien, S. M., Lamanna, N., Kipps, T. J., Flinn, I., Zelenetz, A. D., Burger, J. A., Keating, M., et al. 2015. “A Phase 2 Study of Idelalisib plus Rituximab in Treatment-Naïve Older Patients with Chronic Lymphocytic Leukemia.” Blood 126 (25): 2686-94.

[41] Hotchkiss, R. S., Strasser, A., McDunn, J. E., Swanson, P. E. 2009. "Cell death.” N. Engl. J. Med. 361 (16): 1570-83.

[42] Tsujimoto, Y., Cossman, J., Jaffe, E., and Croce, C. M. 
1985. "Involvement of the bcl-2 Gene in Human Follicular Lymphoma." Science 228 (4706): 1440-3.

[43] Souers, A. J., Leverson, J. D., Boghaert, E. R., Ackler, S. L., Catron, N. D., Chen, J., et al. 2013. "ABT-199, a Potent and Selective BCL-2 Inhibitor, Achieves Antitumor Activity While Sparing Platelets." Nat. Med. 19 (2): 202-8.

[44] Roberts, A. W., Davids, M. S., Pagel, J. M., Kahl, B. S., Puvvada, S. D., Gerecitano, J. F., et al. 2016. "Targeting BCL2 with Venetoclax in Relapsed Chronic Lymphocytic Leukemia." N. Engl. J. Med. 374 (4): 311-22.

[45] Stilgenbauer, S., Eichhorst, B., Schetelig, J., Coutre, S., Seymour, J. F., Munir, T., et al. 2016. "Venetoclax in Relapsed or Refractory Chronic Lymphocytic Leukaemia with 17p Deletion: A Multicentre, Open-Label, Phase 2 Study." Lancet Oncol. 17 (6): 768-78.

[46] Anderson, M. A., Deng, J., Seymour, J. F., Tam, C., Kim,
S. Y., Fein, J., et al. 2016. "The BCL2 Selective Inhibitor Venetoclax Induces Rapid Onset Apoptosis of CLL Cells in Patients via a TP53-Independent Mechanism.” Blood 127 (25): 3215-24.

[47] Jones, J., Choi, M. Y., Mato, A. R., et al. 2016. "Venetoclax (VEN) Monotherapy for Patients with Chronic Lymphocytic Leukemia (CLL) Who Relapsed after or Were Refractory to Ibrutinib or Idelalisib." Blood 128 (22): 637.

[48] Davids, M. S., Roberts, A. W., Seymour, J. F., Pagel, J. M., Kahl, B. S., Wierda, W. G., et al. 2017. "Phase I First-in-Human Study of Venetoclax in Patients with Relapsed or Refractory Non-Hodgkin Lymphoma." J Clin Oncol. 35 (8): 826-33.

[49] Intlekofer, A. M., and Younes, A. 2014. "Precision Therapy for Lymphoma--Current State and Future Directions." Nat. Rev. Clin. Oncol. 11 (10): 585-96. 